UVX 2010 (2011) 153-159

DOI: $10.1051 / \mathrm{uvx} / 2011021$

(C) Owned by the authors, published by EDP Sciences, 2011

\title{
Cristaux et céramiques transparentes comme matériaux scintillateurs pour l'imagerie médicale
}

\author{
B. Viana \\ LCMCP Chimie-Paristech, UMR CNRS 7574 11, Rue Pierre \& Marie Curie, 75231 Paris \\ Cedex 05, France
}

\begin{abstract}
Résumé. Les cristaux et les céramiques transparentes sont très largement utilisés pour la scintillation. Et ceci est plus particulièrement vrai pour l'imagerie médicale. Dans cet article, des travaux sur des matrices d'oxydes mixtes dopés terres rares (cérium et europium) sont rapportés. Un effort tout particulier est porté sur la qualité cristalline pour les cristaux et la transparence pour les céramiques. Pour le volet des propriétés optiques, nous considérons attentivement la réduction de la luminescence persistante tout en conservant un rendement lumineux élevé.
\end{abstract}

\section{INTRODUCTION}

En 1895, Röntgen découvre les rayons $\mathrm{X}$ grâce à la fluorescence visible de $\mathrm{K}_{2} \mathrm{Pt}(\mathrm{CN})_{4}$ déposé sur une plaque de verre. C'est le début de l'utilisation, toujours en expansion, des matériaux scintillateurs. Un matériau scintillateur est capable de convertir l'énergie des radiations ionisantes en lumière visible ou proche UV. Dans la plupart des cas, ces radiations sont des rayons X ou $\gamma$ dont les énergies vont de quelques $\mathrm{keV}$ à plusieurs $\mathrm{MeV}$. La lumière émise par le matériau scintillateur est mesurée grâce à un photomultiplicateur ou des photodiodes à avalanche qui convertissent l'émission visible en signal électrique.

Cet article est consacré aux scintillateurs pour la détection des rayonnements pour les applications médicales. De nombreux matériaux sont développés pour cette application dont les caractéristiques dépendent du rayonnement à détecter, de mode d'acquisition (en comptage, temps de vol) etc. Pour ces applications, la détection directe par un semi-conducteur ( $\mathrm{Si}$ puis $\mathrm{HgI}_{2}$ par exemple pour des énergies plus élevées) ne sont applicables que jusqu'à quelques keV. Cependant ces détecteurs ne présentent de bons rendements qu'à basse température et de plus pour des matériaux de très bonne qualité. Pour la détection de rayonnements plus énergétiques, par exemple les rayons $\mathrm{X}$ d'énergie plus élevée et les rayonnements gamma (voire les neutrons) les matériaux scintillateurs sont inévitables [1-4]. Cet article décrit les avancées dans la réalisation d'oxydes mixtes (céramiques transparentes et cristaux scintillateurs) et les efforts pour limiter la luminescence persistante [5] qui est néfaste lorsque que ces matériaux sont utilisés pour l'imagerie.

\section{APPLICATIONS DES MATÉRIAUX SCINTILLATEURS}

Les utilisations des matériaux scintillateurs sont très variées, citons notamment : (i) le domaine de la sécurité et les contrôles fixes ou mobiles, la vérification du contenu des bagages dans les aéroports, portiques pour le contrôle du contenu de camions etc. (ii) Le contrôle qualité en industrie, (contrôle non

This is an Open Access article distributed under the terms of the Creative Commons Attribution-Noncommercial License 3.0, which permits unrestricted use, distribution, and reproduction in any noncommercial medium, provided the original work is properly cited. 


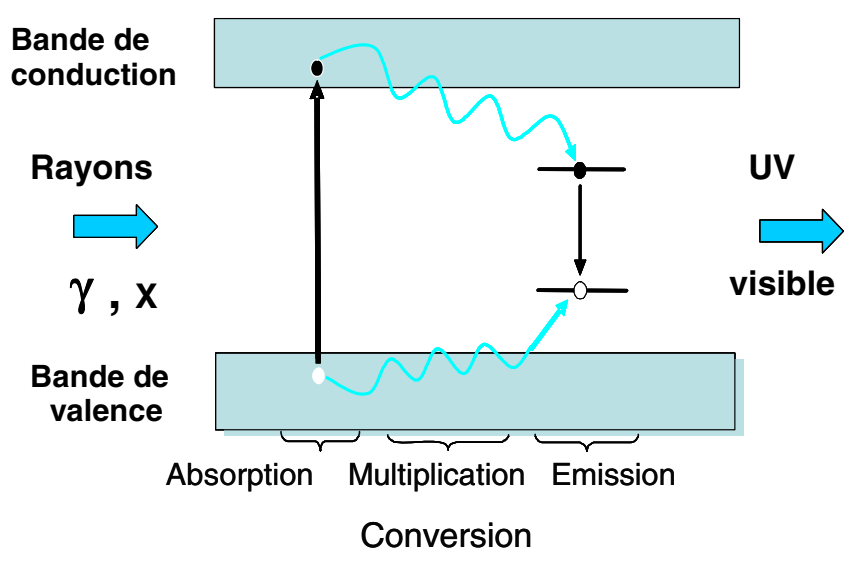

Figure 1. Principe de la scintillation en différentes étapes (absorption des rayonnements très énergétiques, multiplication et émission dans le visible [6].

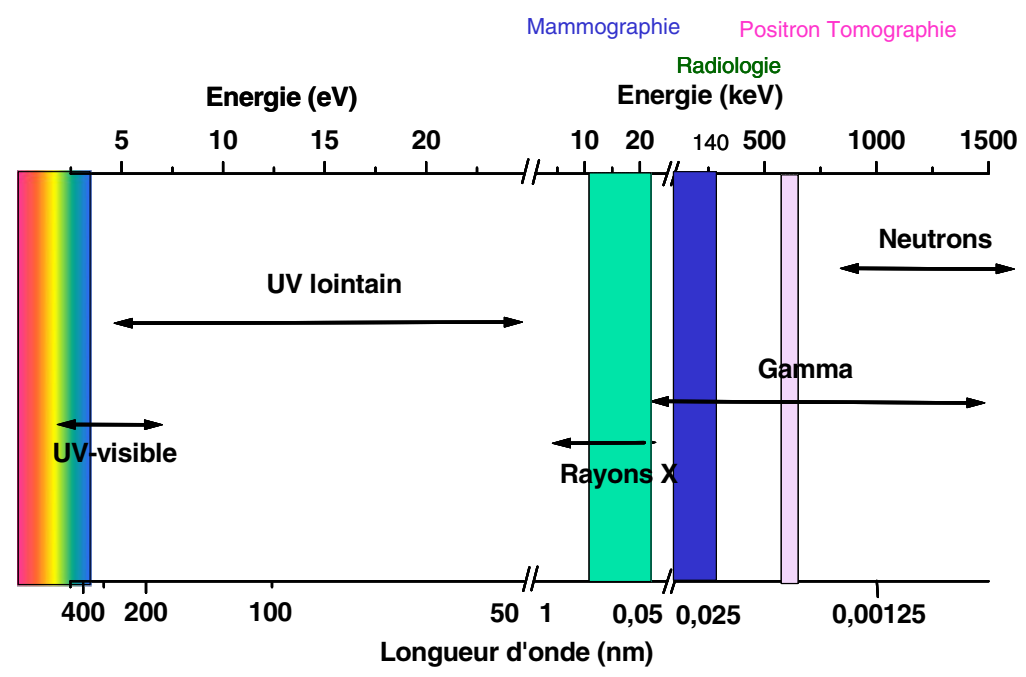

Figure 2. Energie mise en jeu et quelques exemples d'imagerie et des énergies correspondantes.

destructif de matériaux, détection de fissures, de cavités, de défauts). (iii) L'exploration géophysique ; localisation d'hydrocarbures et estimation des conditions d'extraction et de porosité de roches. (iv) La physique des hautes énergies, détection de rayonnements émis lors des collisions de particules...

En imagerie médicale, les systèmes tomographiques sont utilisés à tous les stades de la prise en charge du malade, que ce soit pour le diagnostic de la maladie, la planification de la thérapie ou du geste chirurgical, le guidage des gestes chirurgicaux par l'image pour réaliser des interventions moins invasives, et enfin le suivi d'un traitement thérapeutique.

Détaillons les deux grandes techniques utilisant des scintillateurs :

(i) La tomographie à émission de positrons (TEP) est une technique d'imagerie médicale nucléaire dont l'intérêt est unanimement reconnu dans le monde médical. Dans cette technique, un composé chimique contenant le traceur radioactif émetteur de positron est injecté au patient. Chaque positron se recombinant avec un électron conduit à l'émission colinéaire et de sens opposé de deux photons de $511 \mathrm{keV}$ qui permettent de localiser le point de recombinaison (cf. Fig 3). L'image d'un organe 
spécifique peut ainsi être réalisée. Pour minimiser les doses radioactives, il faut disposer d'un détecteur scintillateur ayant une émission intense sous rayonnement $\gamma$ de $511 \mathrm{keV}$ et un temps de réponse court. Les transitions permises $5 \mathrm{~d}-4 \mathrm{f}$ dans les ions lanthanides, en particulier $\mathrm{Ce}^{3+}$ (dans une matrice $(\mathrm{LuY})_{2} \mathrm{SiO}_{5}$ ) représente un bon compromis entre réponse rapide $40 \mathrm{~ns}$ et un fort rendement lumineux (> 35000 photons/MeV) [7,8].

(ii) La scannographie $\mathrm{X}$ utilise également des scintillateurs. Les différents composants du corps humains (tissus, os,...) absorbent différemment les rayons $\mathrm{X}$ et cela permet d'enregistrer des images des organes internes. Aujourd'hui, la tomographie axiale calculée (Computed Tomography en anglais, ou CT) permet, à partir de plusieurs de ces images, de reconstruire une image en trois dimensions de l'intérieur du corps. La source de rayons X (ou le détecteur) tourne autour du patient, comme on le voit sur la Fig. 3. Les rayons, plus ou moins atténués selon les tissus traversés, sont ensuite détectés par les scintillateurs puis les photodiodes. Les matériaux scintillateurs les plus utilisés aujourd'hui pour la tomographie axiale calculée sont l'oxysulfure de gadolinium dopé au praséodyme (rapide mais dont la transparence n'est pas optimale) et l'oxyde mixte d'yttrium et de gadolinium dopé europium (transparent, mais à réponse plus lente).

La nécessaire rapidité de ces examens et l'acquisition de plusieurs images à des temps rapprochés entraînent des contraintes fortes sur la réponse du matériau scintillateur : toute luminescence rémanente (ou afterglow) perturbera la précision de l'image, en y superposant des informations provenant de l'image précédente. Nous sommes particulièrement intéressés par ce volet au LCMCP-ChimieParistech.
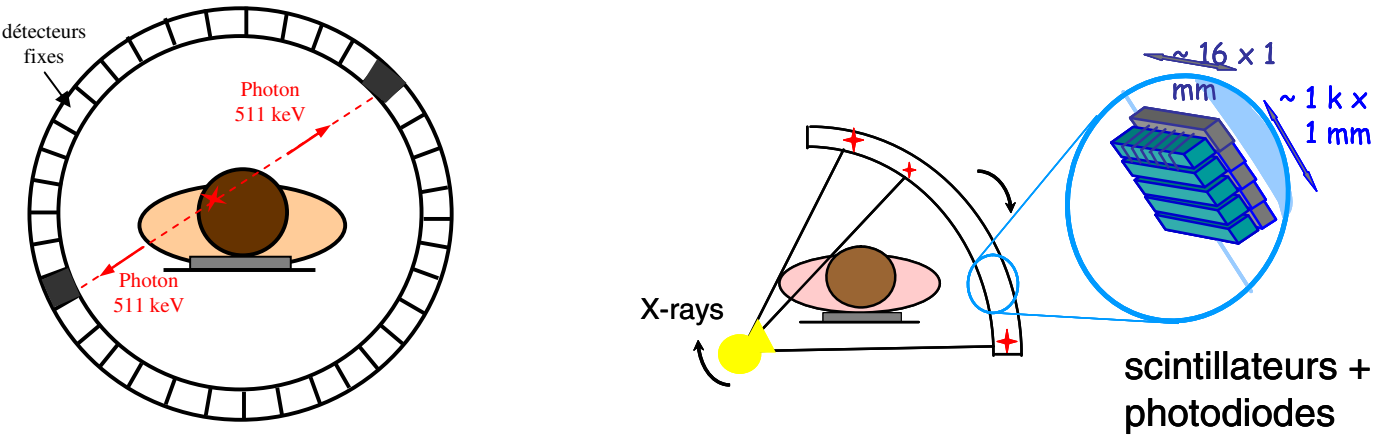

Figure 3. Schéma d'un tomographe à émission de positrons (gauche) et d'un scanner CT.

\section{MÉCANISMES DE SCINTILLATION}

On peut distinguer quatre étapes successives dans les mécanismes de scintillation :

(i) L'absorption du rayonnement électromagnétique, entraînant la création de paires électron-trou (e-t) primaires. Le processus d'absorption le plus important dans le domaine allant du keV e-t quelques $\mathrm{MeV}$ est l'effet photoélectrique. Afin de favoriser cet effet, le produit $\rho Z_{\text {eff }}^{4}$ de la densité $\rho$ du matériau par son numéro atomique, pondéré par la masse de chaque élément, à la puissance 4 doit être élevé. L'effet Compton, correspondant à une absorption partielle de l'énergie, en compétition avec l'effet photoélectrique, est à éviter car il limite le rendement lumineux.

(ii) La multiplication et la thermalisation de ces paires (e-t) qui se poursuit jusqu'à ce qu'il n'y ait plus d'ionisation possible. L'échelle de temps pour ce processus est la picoseconde. Différentes théories ont été développées pour déterminer le nombre de paires (e-t) créées par unité d'énergie absorbée pour un matériau donné et des recherches ont lieu actuellement pour mieux comprendre les mécanismes mis en jeu.

(iii) Le transfert d'énergie aux centres luminescents. Cette étape fondamentale également appelée "migration d'énergie" peut être soit favorable à la scintillation, soit entraîner une extinction de 
la luminescence, soit modifier considérablement la cinétique du processus. Ainsi, les électrons et les trous peuvent former des excitons puis transférer leur énergie aux centres luminescents ou bien encore perdre leur énergie de façon non radiative ou par transfert à un piège (cf. Fig. 8). La complexité de cette étape fait qu'il est très difficile de prédire l'efficacité de cette étape de migration dont le temps caractéristique peut varier entre $10^{-12}$ et $10^{-3}$ secondes. Pour les processus les plus longs, on parle alors de fluorescence retardée ou d' "afterglow".

(iv) La dernière étape est la luminescence de l'ion dopant qui est ici le cérium trivalent (transition 5d-4f) ou l'europium trivalent (transition 4f-4f).

\section{MATÉRIAUX ÉTUDIÉS AU LCMCP}

Pour la tomographie à émission de positron (TEP), deux compositions de cristaux scintillateurs ont été étudiées. Tout d'abord, le LPS $\left(\mathrm{Lu}_{2} \mathrm{Si}_{2} \mathrm{O}_{7}: \mathrm{Ce}^{3+}\right)$ qui a été découvert dans le cadre d'un partenariat industriel [9]. Les avantages attendus de cette composition sont : une basse température de fusion (avantage sur les coûts), la possibilité de meilleurs rendements de cristallisation sur cette composition originale, enfin une meilleure homogénéité en performance de scintillation, attendue de par la structure cristallographique [10]. Par ailleurs, la composition LYSO $\left(\mathrm{Lu}_{1.8} \mathrm{Y}_{0.2} \mathrm{SiO}_{5}: \mathrm{Ce}^{3+}\right)$ que nous avons caractérisée est une composition intéressante car la croissance cristalline de ce composé est plus aisée. Les fabricants de TEP intègrent dans des tomographes de nouvelle génération le scintillateur LYSO : Ce, plus performant en terme de coût de fonctionnement et de capacité de diagnostic. Quant au matériau LPS : Ce, qui est plus délicat à élaborer, seul son comportement en température le rend intéressant pour une application en géophysique. En raison de leurs applications, le développement de ces cristaux à l'échelle industrielle a été réalisé.

Pour ce qui concerne la tomographie CT, les matériaux scintillateurs sur lesquels nous portons toute notre attention sont l'oxysulfure de gadolinium dopé au praséodyme (rapide mais dont la transparence n'est pas optimale) et qui n'est pas présenté ici et l'oxyde mixte de lutécium et de gadolinium dopé europium, transparent, mais à réponse plus lente du fait de la nature de la transition (transition $4 \mathrm{f}-4 \mathrm{f}$ de l'europium).

\section{PRÉPARATION DES MATÉRIAUX}

Pour la partie synthèse des matériaux, celle-ci est effectuée dans un four Czochralski, ainsi que par fusion de zone optique pour les synthèses à petite échelle destinées à la compréhension des phénomènes. Les pièces de plus grandes tailles sont réalisées par Saint Gobain, partenaire industriel.

Pour la réalisation des céramiques, les compositions les plus prometteuses ont été synthétisées sous forme de céramiques transparentes. D'une part un frittage en deux étapes, consistant en un frittage naturel visant à obtenir une porosité fermée suivi d'un frittage sous pression isostatique pour éliminer la porosité résiduelle. Ceci a permis d'obtenir des céramiques transparentes de $\mathrm{Lu}_{2} \mathrm{O}_{3}$ et $\left(\mathrm{Lu}_{0,5} \mathrm{Gd}_{0,5}\right)_{2} \mathrm{O}_{3}$ en collaboration étroite cette fois encore avec notre partenaire industriel (Saint Gobain). Au cours de ces études, nous avons mis en évidence l'importance primordiale de la synthèse de poudres et du processus de frittage sur la qualité finale des céramiques. Les matériaux possèdent une bonne qualité optique et des rendements lumineux en scintillation supérieur à $30000 \mathrm{ph} / \mathrm{Mev}$.

\section{CHALLENGE DE LA LIMITATION DE LA LUMINESCENCE RÉMANENTE}

Pour le LPS:Ce, le matériau scintille à $420 \mathrm{~nm}$ (émission de $\mathrm{Ce}^{3+}$ ), il n'existe pas de luminescence persistante ce qui se traduit par l'absence de bandes en thermoluminescence au voisinage de la température ambiante (zone 300-400 K de la Fig. 6a). Mais malheureusement, ce matériau ne possède pas les propriétés requises en terme de cristallogenèse aisée pour concurrencer le LYSO. Nous avons identifié l'origine de la luminescence persistante (cf fig. 6) et proposé des solutions. Il s'agit, soit d'un 


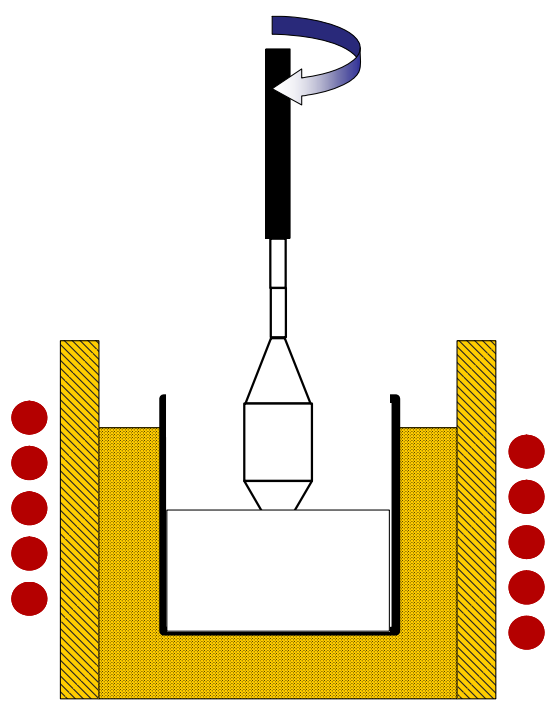

Figure 4. Four Czochralski pour la préparation des monocristaux.
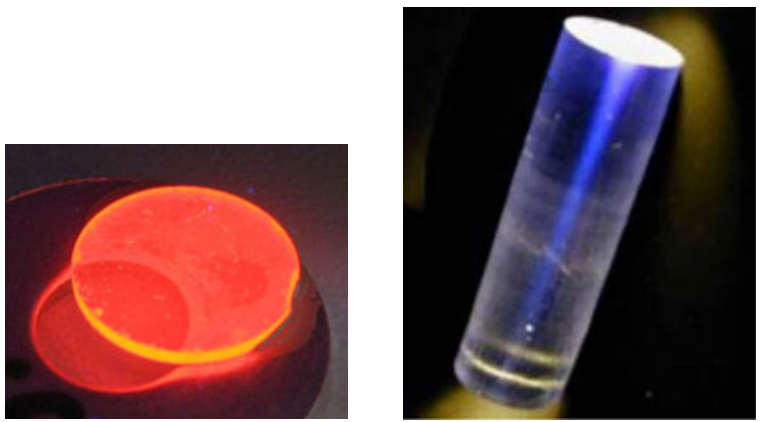

Figure 5. Céramique scintillante de $\mathrm{LuGdO}_{3}$ : Eu obtenue par HIP (gauche) et cristal scintillant de LYSO: Ce (droit) obtenu par la méthode Czochralski. L'émission se situe dans le rouge pour l'europium trivalent (transition $4 \mathrm{f}-4 \mathrm{f}$, temps de vie de $1,5 \mathrm{~ms}$ ) et dans le bleu pour le cérium trivalent (transition 5d-4f, temps de vie $40 \mathrm{~ns}$ ).

traitement thermique pour éliminer des lacunes formées (liées à l'ion O5), soit d'un codopage [11] qui permet de limiter la formation de ces lacunes.

Pour ce qui concerne les céramiques sesquioxydes scintillantes, le dopage par l'ion europium conduit à une luminescence rouge, de temps caractéristique relativement long (voisin de $1,5 \mathrm{~ms}$ ) comparativement au déclin radiatif du cérium. Nous avons montré de plus que le composé mixte $\left(\mathrm{Lu}_{0,5} \mathrm{Gd}_{0,5}\right)_{2} \mathrm{O}_{3}$ :Eu présentait un rendement lumineux élevé et que son temps de vie était plus court, grâce à une occupation préférentielle du site le plus favorable et à une limitation de la distorsion autour de l'ion émetteur [12]. Ceci est visualisé sur la figure 7.

Cette modification de l'occupation des sites a permis de limiter l'afterglow aux temps courts $(<200 \mathrm{~ms})$ et de proposer ces nouvelles céramiques comme scintillateurs pour la tomographie CT.

\section{CONCLUSIONS}

Les scintillateurs ont un grand intérêt pour des multiples applications. Un important effort de recherche reste toutefois nécessaire afin de mieux comprendre les différentes étapes des processus mis en jeux. Pour notre part nous nous intéressons à la recherche de nouveaux matériaux sous toutes les formes possibles et à l'optimisation de leurs propriétés optiques. Signalons que si pour les applications lasers, les céramiques transparentes doivent posséder une qualité optique parfaite, ceci n'est pas indispensable en scintillation pour obtenir un fort rendement lumineux. Ainsi tout récemment $70000 \mathrm{ph} / \mathrm{Mev}$ ont été reporté pour des matériaux sesquioxides dopés europium [13] !.

Pour ce qui concerne la caractérisation des propriétés de rémanence (afterglow), la thermoluminescence est un outil de choix. Cette technique est particulièrement bien appropriée à l'étude des défauts des solides inorganiques (et de leurs mécanismes de formation).

Le schéma de la Fig. 8 visualise les défauts nuisibles aux processus de scintillation et qu'il faut constamment combattre, que ce soit à l'étape de l'élaboration du composé que pour le matériau en 

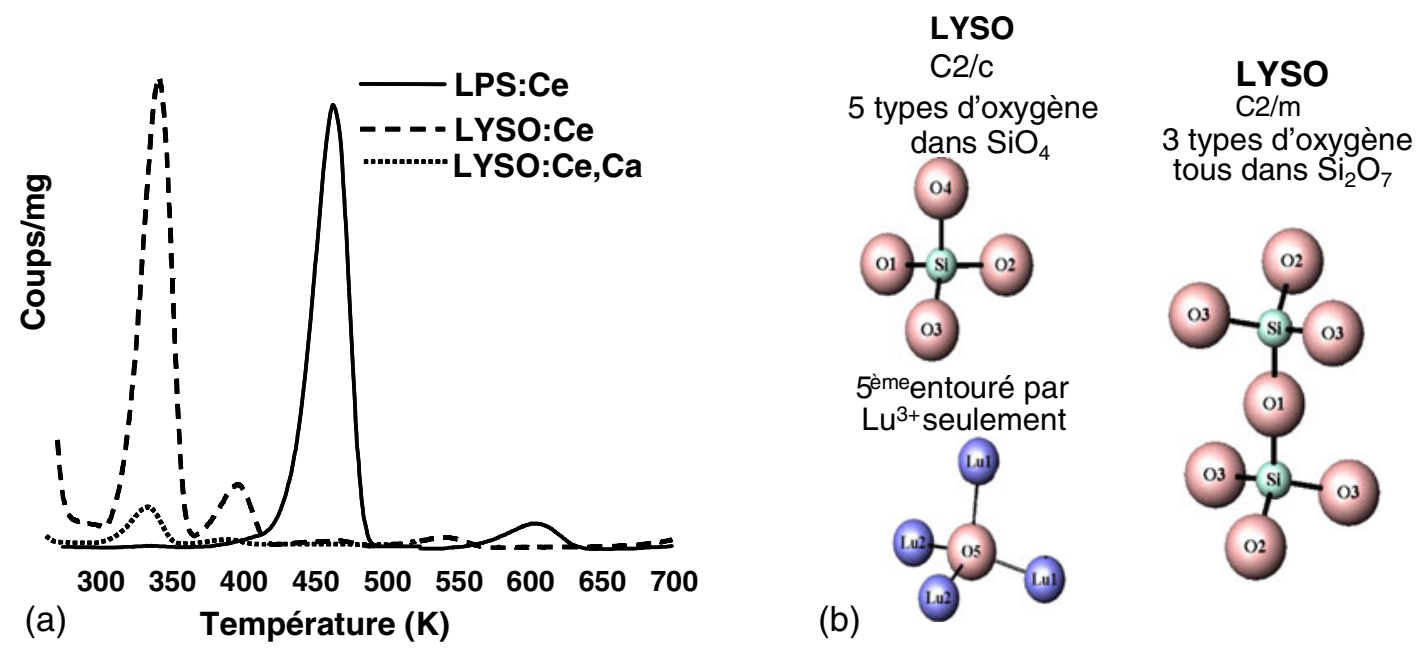

Figure 6. (a) Thermoluminescence des composés LPS :Ce, LYSO :Ce et LYSO :Ce,Ca, composé à faible afterglow [11] ce qui se traduit par une faible intensité au voisinage de la température ambiante. (b) Identification structurale de l'origine des lacunes d'oxygène pour le LYSO (gauche): présence de O5 plus faiblement lié à ses cations (car grandes distances Lu-O5) à l'origine de l'afterglow. Ces groupements ne sont pas présents dans le LPS :Ce (droite).

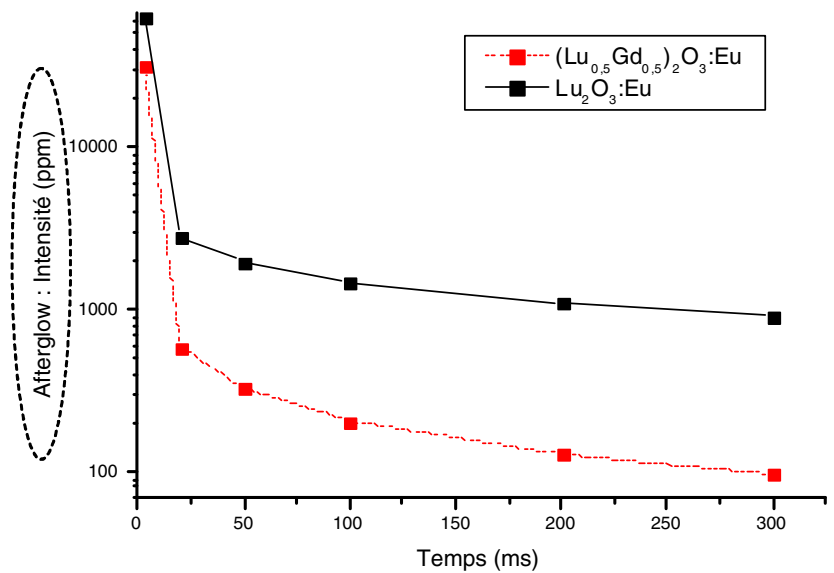

Figure 7. Visualisation de la luminescence persistante (ou afterglow). Celle-ci est très faible dans les composés $\mathrm{LuGdO}_{3}$ : Eu par rapport à $\mathrm{Lu}_{2} \mathrm{O}_{3}[12]$.

service et son vieillissement. Il sera alors possible de mettre au point de nouveaux scintillateurs plus performants ou adaptés à de nouvelles applications.

\section{Remerciements}

L'auteur remercie vivement Saint-Gobain pour le soutien à ces études et L. Pidol, H. Rétot, S. Blahuta, A. Bessière pour leurs travaux au laboratoire sur les cristaux et céramiques comme matériaux scintillateurs pour l'imagerie médicale. 


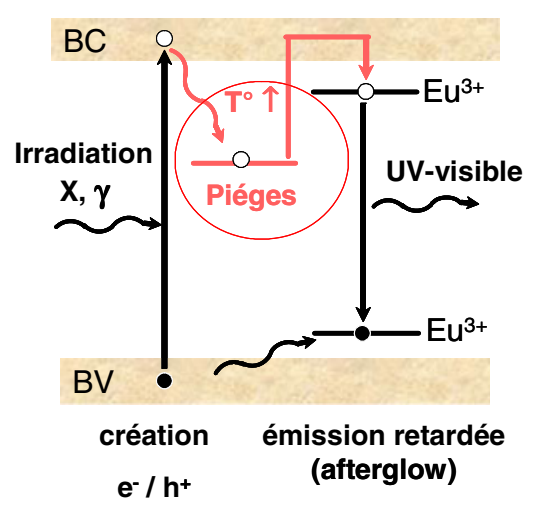

Figure 8. Visualisation des pièges nuisibles en scintillation (pièges à électrons). Afin d'augmenter le signal, il est nécessaire d'éliminer ces pièges responsables de l'afterglow et de la baisse du rendement lumineux.

\section{Références}

[1] P. Dorenbos, L. Pierron L. Dinca L, et al. Journal of Physics-Condensed Matter 153 (2003) 511-520

[2] M.J. Weber dans "selected papers on phosphors, light emitting diodes and scintillators" Ed B.J. Thompson SPIE Vol MS 151 (1998) pp 471-617

[3] W.W. Moses "Scintillator requirements for medical imaging" V Intern. Conf. on Inorganic Scint. and their Applications SCINT 1999 Ed. V.V. Mikhailin, Moscow (2000) pp 11-22

[4] G. Blasse et B.C. Grabmaier "Luminescent Materials" Ed Springer-Verlag (1994) pp 146-193

[5] J.G. Rogers, C.J. Batty IEEE Transactions on Nuclear Science 472 438-445 (2000)

[6] H. Retot, Ph-D thesis, "Elaboration de céramiques transparentes d'oxydes mixtes dopés par des ions lanthanides et caractérisation de leurs propriétés de scintillation "Université P\&M Curie (2009)

[7] L. Pidol, A. Kahn-Harari, B. Viana, et al. IEEE Transactions on Nuclear Science 513 (2004) 1084-1087

[8] I. Valais, C. Michail, S. David, et al., Physica Medica 242 122-125 (2008)

[9] D. Pauwels, N. Le Masson, B. Viana, et al. IEEE Transactions on Nuclear Science 476 (2000) 1787-1790

[10] L. Pidol, O. Guillot-Noel, A. Kahn-Harari et al. Journal of Physics And Chemistry of Solids 674 (2006) 643-650

[11] L. Pidol, B. Viana, B. Ferrand, P. Dorenbos; "Matériau scintillateur dense et rapide à faible luminescence retardée " Brevet PCT/FR2005/050658

[12] H. Retot, S. Blahuta, A. Bessiere, B. Viana, B. LaCourse, E. Mattmann, Chem Mat (soumis) (2010)

[13] N.J. Cherepy; S.A. Payne, et al. (SORMA conference Ann Arbor USA) III 1, 178 (2010). 